\title{
Correlação entre o Índice de Fluxo à Fusão e a Função da Distribuição de Cisão de Cadeia durante a Degradação Termo-Mecânica do Polipropileno
}

\author{
Carlos A. Cáceres C. \\ Programa de Pós-Graduação em Ciência e Engenharia de Materiais, UFSCar \\ Sebastião V. Canevarolo \\ Departamento de Engenharia de Materiais, UFSCar
}

\begin{abstract}
Resumo: Mudanças no Índice de Fluxo à Fusão (MFI) foram correlacionadas com o deslocamento da curva de distribuição de massa molar (MWD) geradas pela degradação termo-mecânica do polipropileno (PP) sujeito a múltiplas extrusões. A degradação foi modelada utilizando-se a Função de Distribuição de Cisão de Cadeia (CSDF), que pode quantificar o deslocamento da curva de distribuição de massa molar com o número de extrusões. O efeito normalmente envolve a redução da massa molar, o que implica em valores positivos da CSDF e redução da viscosidade do estado fundido. $\mathrm{O}$ deslocamento da massa molar média $\left(\log \overline{\mathrm{M}}_{\mathrm{w}(0)}-\log \overline{\mathrm{M}}_{\mathrm{w}(\mathrm{D})}\right)$, calculado a partir das massas molares ponderais médias, antes e após a degradação, se sobrepõe às curvas de CSDF e apresenta uma relação linear com os valores de MFI. O processo de degradação termo-mecânica do polipropileno pode ser mais bem visualizado correlacionando-se curvas de CSDF e os valores de MFI.
\end{abstract}

Palavras-chave: Polipropileno, degradação termo-mecânica, distribuição de massa molar, cisão de cadeia, MFI e CSDF.

\section{Correlation between Melt Flow Index and Chain Scission Distribution Function during the Thermo-Mechanical Degradation of Polypropylene}

Abstract: Changes in the Melt Flow Index were related to the shift in the Molecular Weight Distribution Curve (MWD) pro-
duced by the thermo-mechanical degradation of polypropylene (PP) during multiprocessing. The degradation was modelled
using the Chain Scission Distribution Function (CSDF), which quantifies the shift in the molecular weight distribution curve
as a function of the number of extrusions. The effect is normally the reduction of the molecular weight, which means posi-
tive values for CSDF and reduction of the polymer melt viscosity. The average molecular weight shift (Log $\left.\bar{M}_{\mathrm{w}(0)}-\log \bar{M}_{\mathrm{w}(\mathrm{D})}\right)$
calculated from the average weight molecular weight before and after the degradation overlaps the CSDF curve and shows a
linear relationship with the MFI values. The thermo-mechanical degradation process of polypropylene can be better visual-
ized when CSDF curves are correlated with MFI values.

Keywords: Polypropylene, thermo-mechanical degradation, molecular weight distribution, chain scission, melt flow index and chain scission distribution function.

\section{Introdução}

O polipropileno industrialmente produzido por catalise do tipo Ziegler-Natta, apresenta uma elevada massa molar e uma curva de distribuição de massa molar larga ${ }^{[1]}$. Quando sujeito às solicitações que envolvem altas temperaturas e cisalhamento, típicas do processamento, ele sofre degradação sendo que as cadeias mais longas rompem gerando cadeias menores, decrescendo a massa molar média da resina e ao mesmo tempo estreitando a curva de distribuição de massa molar ${ }^{[1]}$. Este efeito pode ser usado de forma controlada, iniciado com a ajuda de um peróxido orgânico, modificando o polipropileno durante a extrusão. Este processo é uma extrusão reativa e o polímero é conhecido como polipropileno com reologia controlada (CR-PP). O mecanismo aceito para este processo é o de uma iniciação com a formação de radicais livres pela decomposição térmica do peróxido, seguido de abstração de um hidrogênio da cadeia polimérica, quebra da ligação intramolecular C-C e conseqüente cisão da cadeia.

Bremner e Rudin ${ }^{[2]}$ estudaram a variação do MFI para uma série de poliestirenos, polipropilenos e polietilenos de baixa e alta densidade. Neste trabalho mostraram que existe uma relação simples entre os valores de Índice de Fluxo à Fusão (MFI) e a massa molar média. No caso do PP e PS existe uma relação linear entre os valores de MFI e a massa molar ponderal média. 
Uma das variáveis para acompanhar a degradação de polímeros é o número médio de cisões de cadeia $\left(n_{s}\right)$, definida como a relação entre a massa molar numérica média do polímero antes da solicitação termomecânica, dita inicial ou referência $\left(\bar{M}_{\mathrm{n}(0)}\right)$ e após a solicitação com sua conseqüente degradação $\left(\overline{\mathrm{n}}^{(0)}\right)$ e. $)$. Isto pode ser representado matematicamente pela Equação $1^{[3,4]}$.

$$
n_{s}=\frac{\bar{M}_{n(0)}}{\bar{M}_{n(D)}}-1
$$

$\mathrm{Na}$ intenção de melhor entender os efeitos da degradação termo-mecânica em toda a distribuição de massa molar foi proposta $^{[5,6]}$ uma metodologia para calcular a distribuição de número de cisões de cadeia em função da distribuição de massa molar inicial ou de referência. Com tal metodologia é possível obter informações quanto ao motivo da cisão e se esta é afetada pelo comprimento inicial da cadeia polimérica. Esta curva, chamada de Função de Distribuição de Cisão de Cadeia (CSDF), é calculada matematicamente pelas Equações 2 e 3.

$$
\begin{aligned}
& C S D F=\log \left(N_{s}+1\right) \\
& N_{s}=\frac{M W_{(0)}}{M W_{(D)}}-1
\end{aligned}
$$

na Equação 3, $N_{s}$ representa a curva do número de cisões de cadeia no polímero degradado, calculado para cada fração de massa molar que compõe toda a curva de MWD do polímero inicial (ou de referência).

Segundo este modelo a forma da curva de CSDF representa o tipo do processo de degradação. Um processo de degradação termo-mecânico do tipo aleatório pode ser representado por uma curva linear com inclinação zero, indicando que o número de cisões de cadeia é independente da massa molar inicial da cadeia polimérica. Por outro lado o processo de degradação do tipo preferencial mostra uma dependência com a massa molar inicial de tal forma que a CSDF se apresenta como uma curva linear com uma inclinação positiva. Um processo do tipo misto é representado como uma combinação de ambos processos anteriores.

Machado et al ${ }^{[7]}$ estudaram a degradação termomecânica do PP induzida com peróxidos em uma extrusora com duplarosca co-rotativa. A evolução das reações químicas ao longo da extrusora foi caracterizada por medidas reológicas on-line e pela determinação das massas molares de amostras coletadas em válvulas estrategicamente posicionadas ao longo da extrusora. Seguindo o comportamento da Função de Distribuição de Cisão de Cadeia (CSDF) pode-se concluir que o mecanismo de degradação termo-mecânica do PP indica que a cisão de cadeia é dependente do nível de tensão imposta pelos elementos de rosca, temperatura e concentração do peróxido, este último sendo um agente de abstração de hidrogênio e iniciador das reações de cisão de cadeia.

Pinheiro et al. ${ }^{[8]}$ estudaram a degradação termomecânica do HDPE em uma extrusora dupla rosca sob várias condições de processamento. As curvas de CSDF mostram que nos primeiros 3/4 da escala de massa molar os valores de CSDF são negativos, devido à ramificação das cadeias e seu conseqüente aumento na massa molar final. Por outro lado, na região correspondente às cadeias com alta massa molar, os valores de CSDF são positivos. Tal forma da curva de CSDF indica que as cadeias de HDPE mais curtas (com baixa massa molar) ao degradar o fazem gerando preferencialmente ramificações, aumentando sua massa molar original. De forma diversa, as cadeias longas com altas massas molares, tendem preferencialmente à degradar através da cisão de cadeia, com redução da massa molar inicial.

O Índice de Fluxo à Fusão é uma técnica de caracterização reológica que vem sendo usada há mais de quarenta anos para caracterizar a viscosidade no estado fundido de poliolefinas. Devido a sua facilidade de operação, boa repetibilidade dos resultados e baixo custo, acabou sendo aplicada a praticamente todos os demais termoplásticos. Os resultados obtidos por esta técnica dão uma idéia da viscosidade do polímero fundido, normalmente sugerindo a técnica de processamento mais indicada para ele. Apesar de muito conhecida pelos técnicos no setor e largamente empregada o MFI não permite uma visão detalhada, pois não mostra a diversidade de comportamento que diferentes frações do polímero podem apresentar, quantificando apenas o comportamento médio global.

Neste trabalho procurou-se relacionar a forma da curva de CSDF e seus valores médios com os valores de MFI de um polipropileno multiprocessado, na tentativa de mostrar a riqueza de informações que a nova técnica fornece comparada a uma técnica mundialmente reconhecida e corriqueiramente empregada.

\section{Experimental}

\section{Material}

O Polipropileno RP-347 (BRASKEM S.A.), foi usado como recebido e multiprocessado em uma extrusora com dupla-rosca modular co-rotativa e totalmente interpenetrante, Werner \& Pfleiderer modelo ZSK-30 com diâmetro de rosca igual a $30 \mathrm{~mm}$ e comprimento total do barril de $1050 \mathrm{~mm}$. Amostras foram coletadas a cada extrusão. As condições de operação da extrusora, mantidas constantes foram: taxa de alimentação de $5 \mathrm{~kg} / \mathrm{h}$, velocidade de rotação da rosca de $100 \mathrm{rpm}$, perfil constante de temperatura de $240{ }^{\circ} \mathrm{C}$ e perfil de rosca $2 \mathrm{~KB} 45^{[8]}$.

\section{Cromatografia de exclusão por tamanho}

As curvas de distribuição de massa molar (MWD) foram obtidas em um cromatógrafo de exclusão por tamanho, Waters modelo $150 \mathrm{CV}$, com detector por índice de refração e quatro colunas HT (Styragel) recentemente calibradas. As amostras foram solubilizadas a $140{ }^{\circ} \mathrm{C}$ em $1,2,4$ triclorobenzeno (TCB), ambos estabilizados com antioxidante Irganox 1010, filtradas e injetadas no fluxo com vazão de 
$1 \mathrm{~mL} / \mathrm{min}$. As curvas de MWD foram calculadas, utilizando-se o programa Millennium e exportadas como arquivos ASCII para posterior análise.

\section{Função de Distribuição de Cisão de Cadeia (CSDF)}

O cálculo da CSDF foi realizado com o programa CSDF 4. $I^{[6]}$, um macro operando em uma planilha do software Excel (os autores podem fornecer uma cópia a quem se interessar). Os valores da Função Distribuição de Cisão de Cadeia são apresentados em um gráfico como uma função da massa molar da amostra inicial.

\section{Reometria}

O Índice de Fluxo à Fusão (MFI) foi medido à temperatura de $230{ }^{\circ} \mathrm{C}$ e uma carga de $2,16 \mathrm{~kg}$ em um plastômetro fabricado pela Davenport, modelo $\mathrm{N}^{\mathrm{o}} 3$, seguindo a norma ASTM 1238. As curvas de viscosidade em função da taxa de cisalhamento foram obtidas em um reômetro Instron modelo 4467 à mesma temperatura do processo de extrusão, $240{ }^{\circ} \mathrm{C}$.

\section{Resultados e Discussão}

A Figura 1 mostra as curvas de distribuição de massa molar do PP como recebido (virgem) e após re-processado por até 5 vezes na extrusora W\&P ZSK30. As curvas de MWD se deslocam no sentido das baixas massas molares, como consequiência da degradação termomecânica ocorrida a cada extrusão. A Tabela 1 mostra os valores das massas molares numérica e ponderal médias, polidispersão de segunda ordem $\left(\bar{M}_{z} \bar{M}_{w}\right)$, número médio de cisões de cadeia $(n)$ calculado em função da massa molar numérica média $\left(\bar{M}_{n}\right)$, deslocamento do valor de massa molar ponderal média $\left(\log \bar{M}_{w(0)}-\log \bar{M}_{w(D)}\right)$ e o Índice de Fluxo à Fusão (MFI) em função do número de reprocessamentos que o PP sofreu na extrusora.

Durante a primeira extrusão aproximadamente $7 \%$ das cadeias sofrem cisão, apresentando um incremento médio de $6 \%$ a cada extrusão e atingindo a média de uma cisão a cada duas cadeias na quinta extrusão $\left(n_{s} \cong 0,5\right)$. Todas as massas molares médias $\left(\bar{M}_{n}, \bar{M}_{w}, \bar{M}_{z}\right)$ mostram redução, sendo o efeito maior para $\bar{M}_{z}$, que apresenta uma redução média de $12 \%$ a cada extrusão. Estas mudanças provocam pequena variação na polidispersão $\left(\bar{M}_{w} / \bar{M}_{n}\right)$ mas afetam de forma expressiva a polidispersão de segunda ordem $\left(\bar{M}_{z} / \bar{M}_{w}\right)$, indicando a sele- tividade no ataque e redução principalmente das frações de cadeias de PP com alta massa molar.

Relações entre medidas reológicas e MWD indicam que o comportamento da viscosidade a baixas taxas de cisalhamento é uma função da massa molar ponderal média, no entanto, para altas taxas de cisalhamento é a largura da distribuição de massa molar que se torna mais importante ${ }^{[9]}$. A Figura 2 mostra que a viscosidade no estado fundido do PP multiprocessado, em até cinco vezes, reduz com o número de extrusões, apresentando boa concordância com os valores de mas-

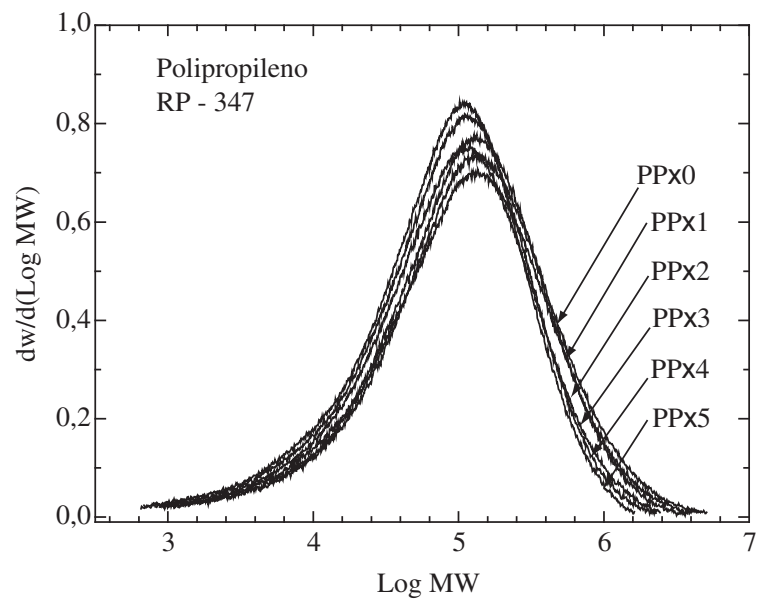

Figura 1. Deslocamento das curvas de distribuição de massa molar devido à degradação termo-mecânica do polipropileno após múltiplas extrusões.

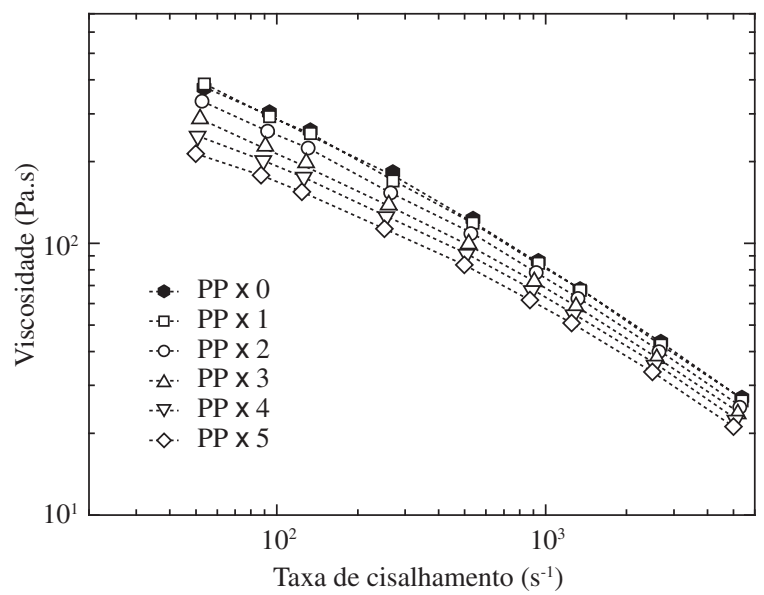

Figura 2. Variação da viscosidade em função da taxa de cisalhamento para o PP virgem e após múltiplas extrusões.

Tabela 1. Massas molares médias $\left(\bar{M}_{n}, \bar{M}_{w}\right)$, polidispersão de segunda ordem $\left(\bar{M}_{z} / \bar{M}_{w}\right)$, número médio de cisões de cadeia $(n)$, diferenças médias $\left(\log \bar{M}_{w(0)}-\log \bar{M}_{w(D)}\right)$ calculados para $\bar{M}_{w}$ e Índice de Fluxo à Fusão (MFI) em função do número de extrusões para polipropileno multiprocessado.

\begin{tabular}{ccccccc}
\hline N $^{\mathbf{e}}$ extr. & $\overline{\boldsymbol{M}}_{\boldsymbol{n}}$ & $\overline{\boldsymbol{M}}_{\boldsymbol{w}}$ & $\overline{\boldsymbol{M}}_{z} \overline{\boldsymbol{M}}_{\boldsymbol{w}}$ & $\boldsymbol{n}_{\boldsymbol{s}}$ & $\boldsymbol{\operatorname { L o g }} \overline{\boldsymbol{M}}_{\boldsymbol{w}(\boldsymbol{\theta})}-\boldsymbol{\operatorname { L o g }} \overline{\boldsymbol{M}}_{\boldsymbol{w}(\boldsymbol{D})}$ & $\mathbf{M F I}(\mathbf{g} / \mathbf{1 0} \mathbf{m i n})$ \\
\hline PPx0* & 41.400 & 233.000 & 3,47 & - & - & 10,0 \\
PPx1 & 38.700 & 217.000 & 3,28 & 0,07 & 0,03 & 11,7 \\
PPx2 & 35.800 & 201.000 & 2,94 & 0,16 & 0,06 & 15,4 \\
PPx3 & 32.800 & 170.000 & 2,76 & 0,26 & 0,14 & 20,9 \\
PPx4 & 30.000 & 158.000 & 2,60 & 0,38 & 0,17 & 27,7 \\
PPx5 & 28.400 & 143.000 & 2,38 & 0,46 & 0,21 & 35,0 \\
\hline
\end{tabular}

*PP virgem 
sa molar ponderal média e a polidispersão de segunda ordem $\left(\bar{M}_{z} / \bar{M}_{w}\right)$, com os valores já apresentados na Tabela 1.

Para um melhor acompanhamento do deslocamento da curva de distribuição de massa molar MWD, conseqüência da degradação termomecânica, calculou-se a Função de Distribuição de Cisão de Cadeia (CSDF). Estas são apresentadas na Figura 3, calculadas a partir da massa molar do polímero virgem (PPx0). Para todas as curvas os valores de CSDF são sempre positivos indicando que a degradação termo-mecânica é exclusivamente por cisão de cadeia. Na primeira extrusão os valores de CSDF são praticamente constantes, independentes da massa molar inicial, indicando um processo de degradação do tipo aleatório. Nas extrusões seguintes a curva começa a apresentar duas regiões com comportamento distinto. Para as frações com baixas massas molares até um valor crítico de massa molar, o processo de degradação continua sendo do tipo aleatório mas, acima deste valor, o processo torna-se dependente de forma crescente da massa molar inicial, ou seja, o processo de degradação muda para um do tipo preferencial.

Já é conhecido na literatura que os valores de MFI mostram uma boa correlação com a massa molar ponderal média $^{[2,10-12]}$. Assim, é de se esperar que transformações que afetem um também induzam mudanças no outro. Seguindo-se nesta lógica é de se esperar que a redução média em $\bar{M}_{w}$, devido a degradação termomecânica, ou seja a diferença média $\left(\log \bar{M}_{w(0)}-\log \bar{M}_{w(D)}\right)$ deva ser acompanhada por um aumento correspondente no Índice de Fluxo à Fusão. A Figura 4 mostra claramente que esta diferença apresenta uma relação linear com os valores de MFI. A diferença média $\left(\log \bar{M}_{w(0)}-\log \bar{M}_{w(D)}\right)$, da forma como apresentada, nada mais é que um caso particular da curva de CSDF quando se analisa exclusivamente um ponto de toda a curva de massas molares, correspondente ao valor de $\mathrm{MW}=\bar{M}_{w}$. Estendendose a análise para abranger toda a faixa de massas molares, ou seja, aquela definida pela curva de CSDF, não se restringe a apenas o valor de massa molar ponderal média. A Figura 5 mostra uma visão tridimensional onde estas três variáveis, massa molar ponderal inicial de cada fração do PP e

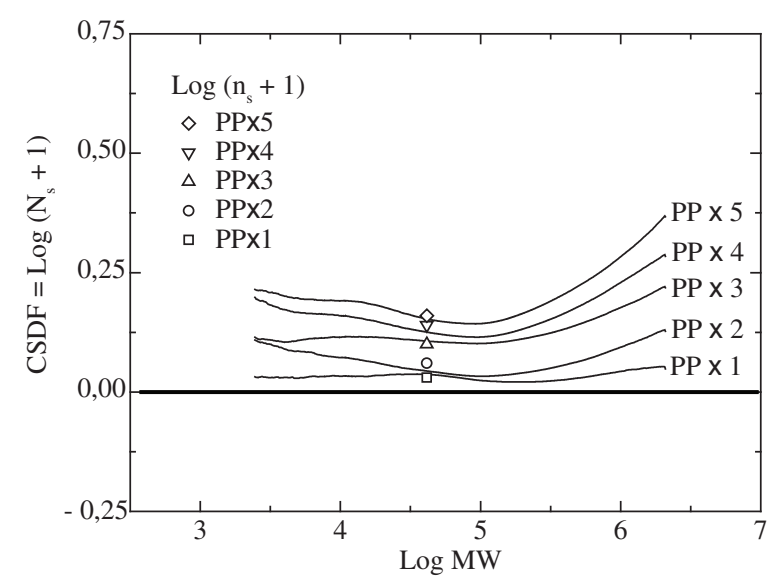

Figura 3. Função da Distribuição de Cisão de Cadeia CSDF como uma função da massa molar inicial do PP multiprocessado. sua correspondência com os valores de distribuição de cisões de cadeia, formando a curva de CSDF são apresentados em função dos valores médios de MFI. Para facilitar a visualização das curvas, evitando sua sobreposição, mudamos o sentido do eixo $\log \left(\overline{\mathrm{M}} \mathrm{W}_{0}\right)$, invertendo a forma convencional de apresentação de uma curva de CSDF. Esta forma de apresentação dos resultados em um gráfico tridimensional permite uma visão mais abrangente das mudanças ocorridas nas várias frações que formam o polímero durante a degradação termomecânica. Todos os comentários já feitos durante a discussão da curva de CSDF apresentada na Figura 3 são válidos aqui, como por exemplo, o fato das curvas de CSDF sempre se deslocarem para cima e que este efeito é mais intenso no lado das maiores massas molares, como consequiência da maior probabilidade de ocorrer cisão nas cadeias mais longas ${ }^{[6]}$. Também pode-se observar que a redução média de $\bar{M}_{w}$ através da diferença média $\left(\log \bar{M}_{w(0)}-\log \bar{M}_{w(D)}\right)$, mostra uma relação linear com a redução da viscosidade, representada pelo aumento nos valores de MFI.

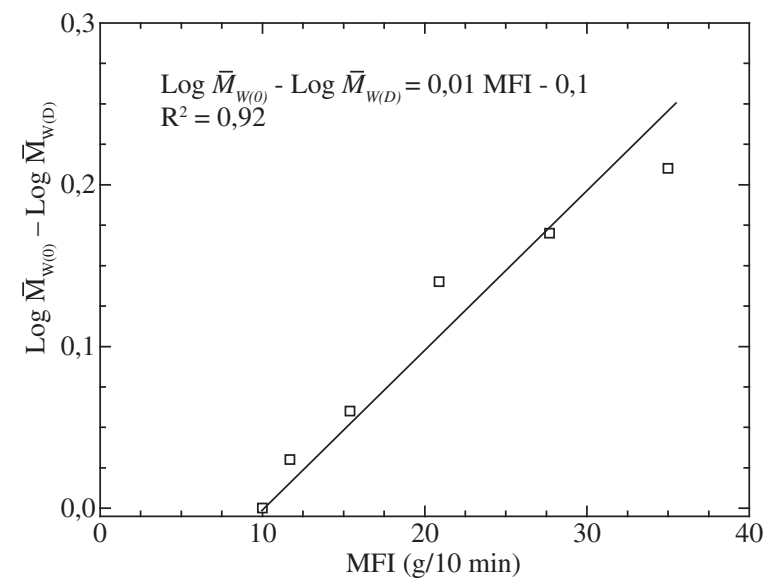

Figura 4. Correlação entre a diferença de massa molar ponderal média $\left(\log \bar{M}_{\mathrm{w}(0)}-\log \bar{M}_{\mathrm{w}(\mathrm{D})}\right)$ e o Índice de Fluxo à Fusão (MFI) para o PP multiprocessado.

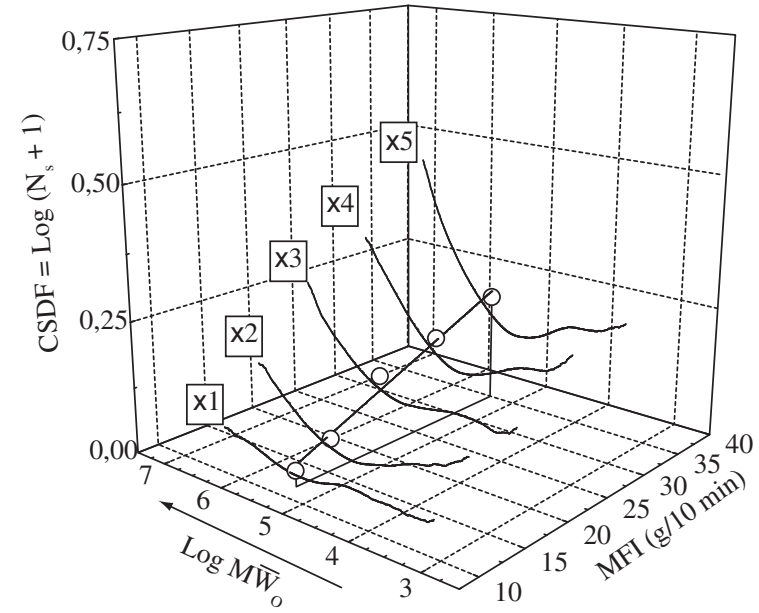

Figura 5. Correlação entre MFI (g/10 min) e as curvas de CSDF para o PP multiprocessado. Como apresentado na figura anterior as diferenças médias $\left(\log \bar{M}_{w(0)}-\log \bar{M}_{w(D)}\right)$ variam linearmente com o MFI. 
É possível concluir que o aumento do MFI, que ocorre durante o processamento do PP devido à cisão de cadeias, é fortemente influenciado pela quebra preferencial das cadeias mais longas. Aproveitando-se de que a curva de CSDF é uma função quantitativa, prevê-se sua utilidade no futuro para um melhor entendimento das mudanças nas curvas de viscosidade com relação à taxa de cisalhamento, ajudando no projeto da melhor forma para a curva de distribuição de massas molares a ser obtida durante a polimerização. Também se prevê a utilidade desta metodologia para o acompanhamento do processo de produção do polipropileno com reologia controlada.

\section{Conclusões}

Curvas de CSDF estão relacionadas a valores do MFI mostrando o efeito de redução da viscosidade do estado fundido devido à cisão de cadeia provocada pela degradação termomecânica do PP multiprocessado. Valores médios $\left(\log \bar{M}_{w(0)}-\log \bar{M}_{w(D)}\right)$, calculados a partir das massas molares ponderais médias, se sobrepõem às curvas de CSDF e apresentam uma relação linear com os valores de MFI. Prevê-se que esta metodologia poderá ser de grande utilidade na caracterização do PP com reologia controlada.

\section{Agradecimentos}

Agradecemos ao $\mathrm{CNPq} / \mathrm{FINEP} / \mathrm{PRONEX}$ pelo apoio financeiro e à CAPES pela bolsa de estudos (C. A. C. C).

\section{Referências Bibliográficas}

1. Vergnes, B.; Berzin, F. - Macromol. Symp., 158, p. 77 (2000).

2. Bremner, T.; Rudin, A. - J. Appl. Polym. Sci., 41, p. 1617 (1990).

3. Reich, L.; Stivala, S. S. - "Elements of polymer degradation", McGraw-Hill, New York (1971).

4. Conley, R. T. - "Thermal Stability of Polymers", Vol. 1, Marcel Dekker, Inc., New York (1970).

5. Canevarolo, S. V. - Polym. Degrad. Stab., 709, p. 71 (2000).

6. Cáceres, C.A.; Canevarolo, S.V.- Polym. Degrad. Stab., 86, p. 437 (2004).

7. Machado, A.; Maia, J.; Canevarolo, S. V.; Covas, J. - Apply. Polym. Sci., 91, p. 2711 (2004).

8. Pinheiro, L. A.; Chinelatto, M. A.; Canevarolo, S. V. Polym. Degrad. Stab., 86, p. 445 (2004).

9. Han, C. D. - "Rheology in Polymer Processing", Academic Press, New York (1976).

10.Hinsken, H.; Moss, S.; Pauquet J. R.; Zweifel, H. - Polym. Degrad. Stab., 34, p. 279 (1991).

11. Vinogradov, G. V.; Malkin, A. Y. - "Rheology of polymer", Mir Publishers, Moscow (1980).

12.Seavey K. C.; Liu, Y. A., Khare N. P.; Bremner, T.; Chyun Chen, C. - Ind. Eng. Chem. Res., 42, p. 5354 (2003).

Enviado: 27/04/06

Reenviado: $18 / 08 / 06$

Aceito: 20/08/06 